\title{
Quality Improvement of Sudanese Petrodiesel Fuel by Furfural
}

\author{
Christina Yacoub Ishak1, Elfatih A. Hassan², Mohamed Ezeldin ${ }^{2,3 *}$ \\ ${ }^{1}$ Department of Chemistry, Faculty of Science, University of Khartoum, Khartoum, Sudan \\ ${ }^{2}$ Department of Chemistry, Faculty of Science, Sudan University of Science and Technology, Khartoum, Sudan \\ ${ }^{3}$ Department of Chemistry, Faculty of Science and Technology, Omdurman Islamic University, Khartoum, Sudan \\ Email: ^Wadalmsna3.com@gmail.com
}

How to cite this paper: Ishak, C.Y., Hassan, E.A. and Ezeldin, M. (2017) Quality Improvement of Sudanese Petrodiesel Fuel by Furfural. American Journal of Analytical Chemistry, 8, 355-369.

https://doi.org/10.4236/ajac.2017.85027

Received: March 4, 2017

Accepted: May 19, 2017

Published: May 22, 2017

Copyright (c) 2017 by authors and Scientific Research Publishing Inc. This work is licensed under the Creative Commons Attribution International License (CC BY 4.0).

http://creativecommons.org/licenses/by/4.0/

\section{(c) (i) Open Access}

\begin{abstract}
The main objective of this project is quality improvement of Sudanese petrodiesel fuel by the use of furfural. The Gas Chromatography Mass Spectrometry GC/MS technique was performed to analyze organic compounds for marked petrodiesel fuel before and after treatment by furfural, physicochemical characteristics of petrodiesel fuel were investigated before and after treatment according to American Society for Testing and Materials (ASTM), characteristics include: density, distillation, cloud point, viscosity, ash content, micro carbon residue, water content, flash point, colour, copper strip corrosion, sulfur content and calculated cetane number. Elements composition of petrodiesel sample has been determined by Inductively coupled plasma (ICP). The furfural showed high ability in extraction of aromatic, cyclo and branched hydrocarbons, a total of 81 organic compounds that exhibit a negative effect on quality of petrodiesel have been removed by furfural. All physicochemical characteristics of petrodiesel fuel were improved within permissible limits assigned by ASTM. The furfural has shown no effect on colour of Sudanese Petrodiesel, which cetane number has increased from 54.46 to 58.36 . The concentration of $\mathrm{Na}, \mathrm{Mg}, \mathrm{Ca}, \mathrm{Fe}, \mathrm{Al}$ and As have been decreased after treatment by furfural, these results have led to decrease of ash content.
\end{abstract}

\section{Keywords}

Sudanese Petrodiesel, Furfural, Khartoum Refinery

\section{Introduction}

The most important property of diesel fuel is cetane number [1], the refineries in the world and researchers in this area are working to improve the cetane number by using environment friendly materials [2]. Cetane number improvers raise the 
cetane number of the fuel. Within a certain range, a higher number can reduce combustion noise and smoke and enhance ease of starting the engine in cold climates [3]. The magnitude of the benefit varies among engine designs and operating modes, ranging from no effect to readily perceptible improvement [3].

2-Ethylhexyl nitrate (EHN) is the most widely used cetane number improver. It is also called octyl nitrate. EHN is thermally unstable and decomposes rapidly at high temperatures in the combustion chamber [4].

The increase in cetane number from a given concentration of EHN varies from one fuel to another. It is greater for a fuel which natural cetane number is already relatively high. The incremental increase gets smaller as more EHN is added, so there is little benefit in exceeding a certain concentration. EHN typically is used in the concentration range from 0.05 to 0.4 percent mass and may yield a three to eight cetane number benefit [5] [6] [7]. In the study, the furfural was used to improve the Sudanese petrodiesel fuel because the furfural is an available material.

\section{Materials and Methods}

\subsection{Materials}

All chemicals used were of analytical reagent grade (AR): Nitric acid, Furfural. Deionized water and Petrodiesel sample.

\subsection{Instrumentation}

\subsubsection{Gas Chromatography Mass Spectrometry}

(Thermo Scientific Co. Thermo GC-TRACE ultra ver.: 5.0, Thermo MS DSQ II). Experimental conditions of GC-MS system were as follows: TR 5-MS capillary standard non-polar column, dimension: 30 Mts, ID: $0.25 \mathrm{~mm}$, Film thickness: $0.25 \mu \mathrm{m}$. Flow rate of mobile phase (carrier gas: $\mathrm{He}$ ) was set at $1.0 \mathrm{ml} / \mathrm{min}$. In the gas chromatography part, temperature program (oven temperature) was $75^{\circ} \mathrm{C}$ raised to $250^{\circ} \mathrm{C}$ at a rise of $5^{\circ} \mathrm{C} / \mathrm{min}$, and held for $30 \mathrm{~min}$.

\subsubsection{Inductively Coupled Plasma}

The analytical determination of metals was carried out by ICP (Inductively Coupled Plasma): ELAN 9000 (Perkin Elmer Sciex Instrument, Concord, Ontario, Canada).

\subsection{Procedures}

The experimental work was conducted at chemistry lab-Omdurman Islamic University, Central lab-University of Khartoum and central lab-Khartoum Refinery, Khartoum and Central Petroleum Laboratories-SUDAN.

\subsubsection{Treatment of Petrodiesel Fuel by Furfural}

Petrodiesel fuel sample $(1000 \mathrm{~mL})$ was treated by furfural $(500 \mathrm{~mL})$ in separating funnel then upper layer was separated and washed with deionized water (1000 $\mathrm{mL})$. 
2.3.2. Physicochemical Properties of Petrodiesel Fuel [8] [9] [10] [11]

The Physicochemical Properties were characterized before and after treatment by furfural according to standard method described by ASTM. Tests includ: Density (D4052), flash point (D93), cloud point (D5773), distillation (D86), kinematic viscosity (D7042), color (D1500), sulfur Content (D5453), water content (D95), copper strip corrosion (D130), carbon Residue (D4530), ash content (D482) and cetane number (D613).

\subsubsection{Gas Chromatography Mass Spectrometry (GC/MS) Analysis of Petrodiesel Fuel}

The GC/MS analysis of petrodiesel fuel before and after treatment by furfural was performed on a GC-MS equipment. The injection volume was $1 \mu \mathrm{l}$ and sample was injected in splitless mode. Finally the sample was run fully at a range of $50-650 \mathrm{~m} / \mathrm{z}$ and the results were compared by using Wiley Spectral library search program [12] [13].

\subsubsection{Characterization of Elements Composition of Petrodiesel Sample by ICP Technique}

The elements composition of petrodiesel fuel was characterized before and after treatment by furfural.

\section{a) Calibration}

The ICP calibration was carried out by external calibration with the blank solution and three working standard solutions $(10,20$ and $30 \mu \mathrm{g} / \mathrm{L})$ for all elements.

\section{b) Preparation of Sample}

About $30 \mathrm{ml}$ of solvent methyl isobutyl-ketone was taken, $5 \mathrm{ml}$ of buffer solution added and then $0.1 \mathrm{~g}$ of iodine weighted and transferred to the solution; 5 $\mathrm{ml}$ of petrodiesel fuel was also added to the solution and the solution finally completed to mark in a $100 \mathrm{ml}$ volumetric flask with methyl isobutyl ketone [14].

\section{Results and Discussion}

\subsection{GC/MS of Petrodiesel before and after Treatment by Furfural}

The organic compounds in petrodiesel sample before and after treatment are shown in Table 1 and Table 2, respectively.

The data obtained from GC/MS revealed that the furfural has shown high ability to extract aromatic, branched and cyclo organic compounds (Table 3 ).

The extracted aromatic, branched and cyclo compounds from petrodiesel sample has confirmed the improvement of its quality, although furfural has extracted Nonadecane yet this has shown high positive effect on $\mathrm{CN}$.

\subsection{The Physicochemical Characteristics of Petrodiesel before and after Treatment by Furfural}

All physicochemical characteristics of petrodiesel sample before and after treatment by furfural were found to be within permissible limits assigned by ASTM Table 4. 
Table 1. Organic compounds of petrodiesel sample before treatment by furfural.

\begin{tabular}{|c|c|c|c|c|c|}
\hline P. No & Compound Name & RT & M. Wt & Area\% & Formula \\
\hline 1 & Octane & 4.614 & 114 & 0.20 & $\mathrm{C} 8 \mathrm{H} 18$ \\
\hline 2 & Ethylcyclohexane & 5.521 & 112 & 0.10 & $\mathrm{C} 8 \mathrm{H} 16$ \\
\hline 3 & 1,1,3-Trimethylcyclohexane & 5.610 & 126 & 0.11 & С9H18 \\
\hline 4 & n-Dodecane & 6.455 & 170 & 0.31 & $\mathrm{C}_{12} \mathrm{H}_{26}$ \\
\hline 5 & 1-Phenyl-3,3-dimethylbutane & 6.667 & 162 & 0.42 & $\mathrm{C} 12 \mathrm{H} 18$ \\
\hline 6 & 1-Ethyl-4-methylcyclohexane & 7.158 & 126 & 0.10 & С9H18 \\
\hline 7 & n-Nonane & 7.592 & 128 & 1.73 & $\mathrm{C} 9 \mathrm{H} 20$ \\
\hline 8 & 1-Ethyl-4-methylcyclohexane & 7.772 & 126 & 012 & $\mathrm{C} 9 \mathrm{H} 18$ \\
\hline 9 & cis-Hexahydroindan & 8.175 & 124 & 0.12 & $\mathrm{C} 9 \mathrm{H} 16$ \\
\hline 10 & Cyclohexane & 8.537 & 126 & 0.28 & С9H18 \\
\hline 11 & 3-Methylnonane & 8.681 & 142 & 0.52 & $\mathrm{C} 10 \mathrm{H} 22$ \\
\hline 12 & (3E)-6-Methyl-3-undecene & 8.920 & 168 & 0.14 & $\mathrm{C} 12 \mathrm{H} 24$ \\
\hline 13 & 1-Pentadecyne & 9.063 & 208 & 0.05 & $\mathrm{C} 15 \mathrm{H} 28$ \\
\hline 14 & 1-Ethyl-2,3-dimethylcyclohexane & 9.211 & 140 & 0.04 & $\mathrm{C} 10 \mathrm{H} 20$ \\
\hline 15 & 1,1,2,3-Tetramethylcyclohexane & 9.374 & 140 & 0.38 & $\mathrm{C} 10 \mathrm{H} 20$ \\
\hline 16 & 4-n-Methylnonane & 9.629 & 142 & 0.21 & $\mathrm{C} 10 \mathrm{H} 22$ \\
\hline 17 & 2-Methyl-nonane & 9.716 & 142 & 0.26 & $\mathrm{C} 10 \mathrm{H} 22$ \\
\hline 18 & 3-Methylnonane & 9.937 & 142 & 0.32 & $\mathrm{C} 10 \mathrm{H} 22$ \\
\hline 19 & 1-Methyl-3-propylcyclohexane & 10.401 & 140 & 0.17 & $\mathrm{C} 10 \mathrm{H} 20$ \\
\hline 20 & Ethylpropylcyclopentane & 10.484 & 140 & 0.10 & $\mathrm{C} 10 \mathrm{H} 20$ \\
\hline 21 & 1,3,5-Trimethylbenzene & 10.565 & 120 & 0.11 & $\mathrm{C} 9 \mathrm{H} 12$ \\
\hline 22 & n-Dodecane & 10.743 & 170 & 0.45 & $\mathrm{C} 12 \mathrm{H} 26$ \\
\hline 23 & 5-Ethyl-2-methylheptane & 10.949 & 142 & 3.82 & $\mathrm{C} 10 \mathrm{H} 22$ \\
\hline 24 & 1-Cyclohexylbutane & 11.668 & 140 & 0.76 & $\mathrm{C} 10 \mathrm{H} 20$ \\
\hline 25 & pentyl-(CAS) Pentylcyclopentane & 11.957 & 140 & 0.32 & $\mathrm{C} 10 \mathrm{H} 20$ \\
\hline 26 & 3,7-Dimethylnonane & 12.098 & 156 & 0.11 & $: \mathrm{C} 11 \mathrm{H} 24$ \\
\hline 27 & trans-Decahydronaphthalene & 12.196 & 138 & 0.11 & $\mathrm{C} 10 \mathrm{H} 18$ \\
\hline 28 & 2,4,6-Trimethyloctane & 12.710 & 156 & 0.12 & $\mathrm{C} 11 \mathrm{H} 24$ \\
\hline 29 & 2-Methyl-5-ethylheptane & 12.821 & 142 & 0.27 & $\mathrm{C} 10 \mathrm{H} 22$ \\
\hline 30 & n-Cetane & 12.924 & 226 & 0.36 & $\mathrm{C} 16 \mathrm{H} 34$ \\
\hline 31 & 3-Methyldecane & 13.043 & 156 & 0.52 & $\mathrm{C} 11 \mathrm{H} 24$ \\
\hline 32 & p-Cymol & 13.255 & 134 & 0.25 & $\mathrm{C} 10 \mathrm{H} 14$ \\
\hline 33 & $\mathrm{n}$-Undecane & 13.808 & 156 & 0.47 & $\mathrm{C} 11 \mathrm{H} 24$ \\
\hline 34 & Decahydro-2-methylnaphthalene & 14.263 & 152 & 5.06 & $\mathrm{C} 11 \mathrm{H} 20$ \\
\hline
\end{tabular}




\section{Continued}

\begin{tabular}{|c|c|c|c|c|c|}
\hline 35 & 2-Butyl-1-octanol & 14.558 & 186 & 0.25 & $\mathrm{C} 12 \mathrm{H} 26 \mathrm{O}$ \\
\hline 36 & 1,2,3,4-Tetramethylbenzene & 14.770 & 134 & 0.37 & $\mathrm{C} 10 \mathrm{H} 14$ \\
\hline 37 & Trans-2-Tridecen-1-ol & 14.949 & 198 & 0.16 & $\mathrm{C} 13 \mathrm{H} 26 \mathrm{O}$ \\
\hline 38 & 1-Cyclohexylpentane & 15.086 & 154 & 0.60 & $\mathrm{C} 11 \mathrm{H} 22$ \\
\hline 39 & Hexylcyclopentane & $15 . .326$ & 154 & 0.35 & $\mathrm{C} 11 \mathrm{H} 22$ \\
\hline 40 & 5-Methylindane & 15.462 & 132 & 0.17 & $\mathrm{C} 10 \mathrm{H} 12$ \\
\hline 41 & 2-Ethylcaproic acid chloride & 15.891 & 162 & 0.15 & $\mathrm{C} 8 \mathrm{H} 15 \mathrm{ClO}$ \\
\hline 42 & 2,3,6,7-Tetramethyloctane & 15.954 & 170 & 0.42 & $\mathrm{C} 12 \mathrm{H} 26$ \\
\hline 43 & 2-Methylundecane & 16.108 & 170 & 0.34 & $\mathrm{C} 12 \mathrm{H} 26$ \\
\hline 44 & 3-Methylundecane & 16.246 & 170 & 0.54 & $\mathrm{C} 12 \mathrm{H} 26$ \\
\hline 45 & 1-methyl-2-pentyl-Cyclohexane & 16.448 & 168 & 0.35 & $\mathrm{C} 12 \mathrm{H} 24$ \\
\hline 46 & 1-(Cyclohexylmethyl)-4-methylcyclohexane & 16.946 & 194 & 0.38 & $\mathrm{C} 14 \mathrm{H} 26$ \\
\hline 47 & (1,1,3,3,5-Pentamethyl-4-hexenyl)benzene & 17.072 & 230 & 0.23 & $\mathrm{C} 17 \mathrm{H} 26$ \\
\hline 48 & n-Heptadecane & 17.267 & 240 & 0.14 & $\mathrm{C} 17 \mathrm{H} 36$ \\
\hline 49 & 3,6-Dimethylundecane & 17.408 & 140 & 4.98 & $\mathrm{C} 13 \mathrm{H} 28$ \\
\hline 50 & 1,2,3,4-tetrahydro-2-methyl-naphthalene & 17.785 & 146 & 0.82 & $\mathrm{C} 11 \mathrm{H} 14$ \\
\hline 51 & 10-Heneicosene & 17.954 & 294 & 0.32 & $\mathrm{C} 21 \mathrm{H} 42$ \\
\hline 52 & 4-Methylpentylcyclohexane & 18.063 & 168 & 0.08 & $\mathrm{C} 12 \mathrm{H} 24$ \\
\hline 53 & 3-Tetradecene & 18.223 & 196 & 0.28 & $\mathrm{C} 14 \mathrm{H} 28$ \\
\hline 54 & 6-Methyldodecane & 18.543 & 114 & 0.45 & $\mathrm{C} 13 \mathrm{H} 28$ \\
\hline 55 & 1-Ethyl-1-methylindane & 18.637 & 160 & 0.19 & $\mathrm{C} 12 \mathrm{H} 16$ \\
\hline 56 & 11-Benzylheneicosane & 18.933 & 386 & 0.11 & $\mathrm{C} 28 \mathrm{H} 50$ \\
\hline 57 & 4-Methyldodecane & 18.988 & 184 & 0.21 & $\mathrm{C} 13 \mathrm{H} 28$ \\
\hline 58 & 2,10-Dimethylundecane & 19.128 & 184 & 0.13 & $\mathrm{C} 13 \mathrm{H} 28$ \\
\hline 59 & 1,2,3,4-tetrahydro-6-methyl-Naphthalene & 19.266 & 146 & 0.42 & $\mathrm{C} 11 \mathrm{H} 14$ \\
\hline 60 & 2,7,10-Trimethyldodecane & 19.457 & 212 & 0.57 & $\mathrm{C} 15 \mathrm{H} 32$ \\
\hline 61 & 3,7,11,15-Tetramethylhexadecanol & 19.525 & 298 & 0.34 & $\mathrm{C} 20 \mathrm{H} 42 \mathrm{O}$ \\
\hline 62 & Heptadecyl octanoate & 19.714 & 382 & 0.20 & $\mathrm{C} 25 \mathrm{H} 50 \mathrm{O} 2$ \\
\hline 63 & 2-Pentadecyl-1,3-dioxolan-4-yl)methyl acetate & 19.871 & 356 & 0.08 & $\mathrm{C} 21 \mathrm{H} 40 \mathrm{O} 4$ \\
\hline 64 & 1,6-Dimethylindane & 19.941 & 146 & 0.09 & $\mathrm{C} 11 \mathrm{H} 14$ \\
\hline 65 & 2-methyl-4-(2-methylpropyl)-Cyclopentanone & 20.026 & 154 & 0.20 & $\mathrm{C} 10 \mathrm{H} 18 \mathrm{O}$ \\
\hline 66 & $\mathrm{n}$-Tridecane & 20.373 & 184 & 5.79 & $\mathrm{C} 13 \mathrm{H} 28$ \\
\hline 67 & Isododecane & 20.833 & 170 & 0.32 & $\mathrm{C} 12 \mathrm{H} 26$ \\
\hline
\end{tabular}




\section{Continued}

\begin{tabular}{|c|c|c|c|c|c|}
\hline 68 & 6-methyl-Tridecane & 21.008 & 198 & 0.51 & $\mathrm{C} 14 \mathrm{H} 30$ \\
\hline 69 & 1,2,3,4-Tetrahydro-2,6-dimethylnaphthalene & 21.124 & 160 & 0.11 & $\mathrm{C} 12 \mathrm{H} 16$ \\
\hline 70 & (2,2-dimethyl-1-methylenepropyl)-benzene & 21.338 & 160 & 0.10 & $\mathrm{C} 12 \mathrm{H} 16$ \\
\hline 71 & 1-Ethyl-1-methylindane & 21.436 & 160 & 0.13 & $\mathrm{C} 12 \mathrm{H} 16$ \\
\hline 72 & 3,5-Dimethyldodecane & 21.568 & 198 & 0.39 & $\mathrm{C} 14 \mathrm{H} 30$ \\
\hline 73 & 1,4-Dicyclohexylbutan & 21.637 & 122 & 0.13 & $\mathrm{C} 16 \mathrm{H} 30$ \\
\hline 74 & Decylcyclopentane & 21.752 & 120 & 0.34 & C15H30 \\
\hline 75 & 6-Methyltridecan & 21.828 & 198 & 0.19 & $\mathrm{C} 14 \mathrm{H} 30$ \\
\hline 76 & 5-Methyltetradecane & 21.968 & 212 & 0.28 & $\mathrm{C} 15 \mathrm{H} 32$ \\
\hline 77 & 2,3-Dimethyldodecane & 22.112 & 198 & 0.59 & $\mathrm{C} 14 \mathrm{H} 30$ \\
\hline 78 & n-Pentadecane & 22.302 & 212 & 0.32 & $\mathrm{C} 15 \mathrm{H} 32$ \\
\hline 79 & 3,5-Dimethyldodecane & 22.465 & 198 & 0.81 & $\mathrm{C} 14 \mathrm{H} 30$ \\
\hline 80 & 2,7,10-Trimethyldodecane & 22.907 & 212 & 0.37 & $\mathrm{C} 15 \mathrm{H} 32$ \\
\hline 81 & (7E)-17-Chloro-7-heptadecene & 23.159 & 272 & 5.40 & $\mathrm{C} 17 \mathrm{H} 33 \mathrm{Cl}$ \\
\hline 82 & n-Tetradecane & 23.228 & 198 & 0.27 & $\mathrm{C} 14 \mathrm{H} 30$ \\
\hline 83 & 2,6,10-Trimethylpentadecane & 23.885 & 254 & 0.28 & $\mathrm{C} 18 \mathrm{H} 38$ \\
\hline 84 & Methyl phenyl pentanal & 23.957 & 174 & 0.14 & $\mathrm{C} 12 \mathrm{H} 14 \mathrm{O}$ \\
\hline 85 & 4-(4-propyl)bicyclo hexyl & 24.192 & 298 & 0.23 & $\mathrm{C} 22 \mathrm{H} 34$ \\
\hline 86 & Do decane 2-cyclo hexane & 24,417 & 252 & 0.81 & $\mathrm{C} 18 \mathrm{H} 36$ \\
\hline 87 & Tetradecane & 24.650 & 212 & 0.33 & $\mathrm{C} 15 \mathrm{H} 32$ \\
\hline 88 & Tetradecane & 24.750 & 198 & 0.82 & $\mathrm{C} 14 \mathrm{H} 30$ \\
\hline 89 & 2-N-Butyl-8-N-hexyldecahydronaphthalene & 24.875 & 278 & 0.31 & $\mathrm{C} 20 \mathrm{H} 38$ \\
\hline 90 & 3-Metyl Tetradecane & 24.975 & 212 & 0.23 & $\mathrm{C} 15 \mathrm{H} 32$ \\
\hline 91 & 1-H-hydro indeneocta & 24.975 & 208 & 0.36 & $\mathrm{C}_{15} \mathrm{H}_{28}$ \\
\hline 92 & 2,2,4,4,7,7-methyl-trans heta & 25.136 & 212 & 0.30 & $\mathrm{C}_{15} \mathrm{H}_{32}$ \\
\hline 93 & Penta decane nona decane & 25.784 & 268 & 5.00 & $\mathrm{C}_{19} \mathrm{H}_{40}$ \\
\hline 94 & 1-methyl-(6-ethyl-3-decyloxy)-1-silay-dohexane & 25.961 & 298 & 0.13 & $\mathrm{C}_{18} \mathrm{H}_{38}$ \\
\hline 95 & Diteradodecyl Bicyclo(4-1)heproan-2-1Disulfide & 26.5 & 402 & 0.12 & $\mathrm{C}_{24} \mathrm{H}_{50} \mathrm{~S}_{2}$ \\
\hline 96 & 1-phenyl 1-endo & 26.6 & 188 & 0.11 & $\mathrm{C}_{24} \mathrm{H}_{16} \mathrm{O}$ \\
\hline 97 & 5-(2-methyl lapropyl)Ranane & 26.733 & 184 & 0.11 & $\mathrm{C}_{13} \mathrm{H}_{36}$ \\
\hline 98 & 4-cycohexyl Undecane & 26.925 & 238 & 0.14 & $\mathrm{C}_{17} \mathrm{H}_{34}$ \\
\hline 99 & 4-methyl Tetradecane & 27.133 & 212 & 0.42 & $\mathrm{C}_{15} \mathrm{H}_{32}$ \\
\hline
\end{tabular}




\section{Continued}

\begin{tabular}{|c|c|c|c|c|c|}
\hline 100 & 2-methyl Tridecane & 27.225 & 198 & 0.27 & $\mathrm{C}_{14} \mathrm{H}_{30}$ \\
\hline 101 & Eicosane & 27.342 & 282 & 0.20 & $\mathrm{C}_{20} \mathrm{H}_{42}$ \\
\hline 102 & 3-methyl Pentade & 27.408 & 226 & 0.11 & $\mathrm{C}_{16} \mathrm{H}_{34}$ \\
\hline 103 & 14-methyl-8-hetadecane-1 & 27.525 & 254 & 0.25 & $\mathrm{C}_{17} \mathrm{H}_{34}$ \\
\hline 104 & 1-one4-methyl-4phenyl-2-cyclohexan & 27.633 & 186 & 0.13 & $\mathrm{C}_{13} \mathrm{H}_{14} \mathrm{O}$ \\
\hline 105 & 3-5,24-trimethyl Tetra eontane & 27.750 & 604 & 0.10 & $\mathrm{C}_{43} \mathrm{H}_{88}$ \\
\hline 106 & Hexadecane & 28.042 & 220 & 0.19 & $\mathrm{C}_{16} \mathrm{H}_{34}$ \\
\hline 107 & 3,6-di methylUndecane & 28.300 & 184 & 5.20 & $\mathrm{C}_{13} \mathrm{H}_{28}$ \\
\hline 108 & 3-cydohexyl, dodecane & 29.417 & 252 & 0.58 & $\mathrm{C}_{18} \mathrm{H}_{36}$ \\
\hline 109 & Octodecane & 29.642 & 254 & 0.61 & $\mathrm{C}_{18} \mathrm{H}_{38}$ \\
\hline 110 & 3-methyl hexa decane & 29.750 & 240 & 0.32 & $\mathrm{C}_{17} \mathrm{H}_{36}$ \\
\hline 111 & Hepta decane & 29.933 & 240 & 0.30 & $\mathrm{C}_{17} \mathrm{H}_{36}$ \\
\hline 112 & 2,6,10,14 Tetra methyl Pema decane & 30.650 & 268 & 4.98 & $\mathrm{C}_{19} \mathrm{H}_{40}$ \\
\hline 113 & 9,Octyl Hepta decane & 30.750 & 352 & 0.98 & $\mathrm{C}_{17} \mathrm{H}_{52}$ \\
\hline 114 & 3-methyl-Hepta decane & 32.017 & 254 & 0.31 & $\mathrm{C}_{18} \mathrm{H}_{38}$ \\
\hline 115 & Heptadecane & 32.200 & 240 & 0.18 & $\mathrm{C}_{17} \mathrm{H}_{36}$ \\
\hline 116 & 2,6,10,10,14, Tetra methyl Hexadecane & 32.883 & 282 & 4.67 & $\mathrm{C}_{20} \mathrm{H}_{42}$ \\
\hline 117 & Hexadecane & 33.050 & 226 & 0.65 & $\mathrm{C}_{16} \mathrm{H}_{34}$ \\
\hline 118 & Octodecane & 34.200 & 254 & 0.38 & $\mathrm{C}_{18} \mathrm{H}_{38}$ \\
\hline 119 & Heneicosane & 35.008 & 296 & 4.65 & $\mathrm{C}_{21} \mathrm{H}_{44}$ \\
\hline 120 & 4-cyclohexyl Tridecane & 36.242 & 266 & 0.26 & $\mathrm{C}_{19} \mathrm{H}_{38}$ \\
\hline 121 & Eicosane & 36.417 & 282 & 0.37 & $\mathrm{C}_{20} \mathrm{H}_{42}$ \\
\hline 122 & Heneicosane & 37.025 & 296 & 4.01 & $\mathrm{C}_{21} \mathrm{H}_{44}$ \\
\hline 123 & Heneicosane & 38.950 & 296 & 3.58 & $/ /$ \\
\hline 124 & Cudo Hexane & 40.048 & 238 & 0.15 & $\mathrm{C}_{17} \mathrm{H}_{34}$ \\
\hline 125 & Henei cosine & 40.338 & 296 & 0.13 & $\mathrm{C}_{21} \mathrm{H}_{44}$ \\
\hline 126 & Pentacosane & 40.756 & 352 & 2.81 & $\mathrm{C}_{25} \mathrm{H}_{52}$ \\
\hline 127 & Henei cosine & 40.842 & 296 & 0.10 & $\mathrm{C}_{21} \mathrm{H}_{44}$ \\
\hline 128 & Heneicosane & 42.517 & & 2.10 & $/ /$ \\
\hline 129 & Pentacosane & 44.207 & 352 & 1.35 & $\mathrm{C}_{25} \mathrm{H}_{52}$ \\
\hline 130 & Henei cosine & 45.942 & 296 & 0.85 & $\mathrm{C}_{21} \mathrm{H}_{44}$ \\
\hline 131 & Hexa cosane & 48.007 & 366 & 0.49 & $\mathrm{C}_{26} \mathrm{H}_{54}$ \\
\hline 132 & Hexa cosane & 50.548 & 366 & 0.37 & $\mathrm{C}_{26} \mathrm{H}_{54}$ \\
\hline
\end{tabular}


Table 2. Organic compounds of petrodiesel sample after treatment by furfural.

\begin{tabular}{|c|c|c|c|c|c|}
\hline P. NO & Compound Name & RT & M. Wt & Erea\% & Formula \\
\hline 1 & Octane & 5.517 & 114 & 0.21 & $\mathrm{C}_{8} \mathrm{H}_{18}$ \\
\hline 2 & Carboxal dehyde furan & 6.683 & 96 & 3.67 & $\mathrm{C}_{5} \mathrm{H}_{4} \mathrm{O}_{2}$ \\
\hline 3 & 5_methyl Undecane & 7.592 & 170 & 0.20 & $\mathrm{C}_{12} \mathrm{H}_{26}$ \\
\hline 4 & 3_methyl Octane & 7.825 & 128 & 0.35 & $\mathrm{C}_{9} \mathrm{H}_{20}$ \\
\hline 5 & 1_ethyl_4_methyl-Cyclohexane & 8.317 & 126 & 0.11 & $\mathrm{C}_{9} \mathrm{H}_{18}$ \\
\hline 6 & Nonane & 8.808 & 128 & 1.68 & $\mathrm{C}_{9} \mathrm{H}_{20}$ \\
\hline 7 & 3_methyl nonane & 9.958 & 142 & 0.46 & $\mathrm{C}_{10} \mathrm{H}_{22}$ \\
\hline 8 & 4_methyl nonane & 10.942 & 142 & 0.32 & $\mathrm{C}_{10} \mathrm{H}_{22}$ \\
\hline 9 & 2_methyl n nane & 11.025 & 142 & 0.21 & $\mathrm{C}_{10} \mathrm{H}_{22}$ \\
\hline 10 & 3_methyl nonane & 11.258 & 142 & 0.27 & $\mathrm{C}_{10} \mathrm{H}_{22}$ \\
\hline 11 & 1,3,5_trimethyl benzene & 12.067 & 120 & 0.31 & $\mathrm{C}_{9} \mathrm{H}_{22}$ \\
\hline 12 & Decane & 12.283 & 142 & 4.41 & $\mathrm{C}_{10} \mathrm{H}_{22}$ \\
\hline 13 & 5_methyl_2_methyl Heptanes & 13.033 & 142 & 0.81 & $\mathrm{C}_{10} \mathrm{H}_{22}$ \\
\hline 14 & Butyl cycloheptane & 13.308 & 140 & 0.34 & $\mathrm{C}_{10} \mathrm{H}_{20}$ \\
\hline 15 & 5_Methyl decane & 14.200 & 156 & 0.21 & $\mathrm{C}_{11} \mathrm{H}_{29}$ \\
\hline 16 & 2_methyl_5_ethyl heptanes & 14.300 & 142 & 0.33 & $\mathrm{C}_{10} \mathrm{H}_{22}$ \\
\hline 17 & Hexadecane & 14.425 & 226 & 0.53 & $\mathrm{C}_{16} \mathrm{H}_{34}$ \\
\hline 18 & 3_Methyl decane & 14.333 & 156 & 0.24 & $\mathrm{C}_{11} \mathrm{H}_{24}$ \\
\hline 19 & Undecane & 15.333 & 156 & 5.60 & $\mathrm{C}_{11} \mathrm{H}_{24}$ \\
\hline 20 & Tridecanaldehyde & 16.467 & 198 & 0.61 & $\mathrm{C}_{13} \mathrm{H}_{20} \mathrm{O}$ \\
\hline 21 & Pentyl cyclohexane & 13.708 & 154 & 0.45 & $\mathrm{C}_{11} \mathrm{H}_{22}$ \\
\hline 22 & 1,1_Bis(iso pentyloxy)hexadecane & 17.492 & 398 & 0.35 & $\mathrm{C}_{26} \mathrm{H}_{54} \mathrm{O}_{2}$ \\
\hline 23 & 2_methyl undecane & 17.633 & 170 & 0.65 & $\mathrm{C}_{12} \mathrm{H}_{26}$ \\
\hline 24 & 3,8_methyl undecane & 17.842 & 170 & 0.32 & $\mathrm{C}_{12} \mathrm{H}_{26}$ \\
\hline 25 & Tridecane & 18.783 & 184 & 5.96 & $\mathrm{C}_{13} \mathrm{H}_{28}$ \\
\hline 26 & 3,6_dimethyl undecane & 19.167 & 184 & 0.72 & $\mathrm{C}_{13} \mathrm{H}_{28}$ \\
\hline 27 & Tridecane & 21.742 & 184 & 5.88 & $\mathrm{C}_{13} \mathrm{H}_{28}$ \\
\hline 28 & 2,7,10_trimethyl dodecane & 23.842 & 212 & 0.46 & $\mathrm{C}_{15} \mathrm{H}_{28}$ \\
\hline 29 & Bentadecane & 23.483 & 212 & 0.81 & $\mathrm{C}_{15} \mathrm{H}_{32}$ \\
\hline 30 & ISO tetrodecane & 24.525 & 198 & 6.45 & $\mathrm{C}_{14} \mathrm{H}_{30}$ \\
\hline 31 & 2,6,10,14_tetremethyl hexadecane & 26.125 & 282 & 1.45 & $\mathrm{C}_{20} \mathrm{H}_{42}$ \\
\hline 32 & N_pentadecane & 27.142 & 212 & 6.08 & $\mathrm{C}_{15} \mathrm{H}_{32}$ \\
\hline 33 & N_hexadecane(cetane) & 29.625 & 226 & 6.59 & $\mathrm{C}_{16} \mathrm{H}_{34}$ \\
\hline 34 & Heptadecane & 31.967 & 240 & 6.15 & $\mathrm{C}_{14} \mathrm{H}_{36}$ \\
\hline
\end{tabular}




\section{Continued}

\begin{tabular}{llllll}
\hline 35 & 2,6,10,14_tetramethyl p; entadecane & 32.067 & 268 & 1.44 & $\mathrm{C}_{19} \mathrm{H}_{40}$ \\
36 & Octadecane & 34.200 & 254 & 5.73 & $\mathrm{C}_{17} \mathrm{H}_{36}$ \\
37 & 2,6,10,10_tetramethyl Hexadecane & 34.367 & 282 & 0.92 & $\mathrm{C}_{20} \mathrm{H}_{42}$ \\
38 & Nonanedecane & 36.325 & 268 & 5.90 & $\mathrm{C}_{19} \mathrm{H}_{40}$ \\
39 & Eicosane & 38.342 & 282 & 5.27 & $\mathrm{C}_{20} \mathrm{H}_{42}$ \\
40 & Heneicosane & 40.267 & 296 & 5.07 & $\mathrm{C}_{21} \mathrm{H}_{44}$ \\
41 & Heneicosane & 40.267 & 296 & 5.07 & $\mathrm{C}_{21} \mathrm{H}_{44}$ \\
42 & Pentacosane & 42.108 & 352 & 4.08 & $\mathrm{C}_{25} \mathrm{H}_{52}$ \\
43 & Pentacosane & 45.875 & 352 & 3.14 & $\mathrm{C}_{25} \mathrm{H}_{52}$ \\
44 & Pentacosane & 45.575 & 352 & 2.08 & $\mathrm{C}_{25} \mathrm{H}_{52}$ \\
45 & Pentacosane & 47.200 & 352 & 1.32 & $\mathrm{C}_{25} \mathrm{H}_{52}$ \\
46 & Pentacosane & 48.767 & 352 & 0.71 & $\mathrm{C}_{25} \mathrm{H}_{52}$ \\
47 & Pentacosane & 50.283 & 352 & 0.55 & $\mathrm{C}_{25} \mathrm{H}_{52}$ \\
48 & Pentacosane & 51.742 & 352 & 0.32 & $\mathrm{C}_{25} \mathrm{H}_{52}$ \\
49 & Hexacosane & 53.150 & 366 & 0.24 & $\mathrm{C}_{26} \mathrm{H}_{54}$ \\
50 & Tetracontane & 54.508 & 563 & 0.14 & $\mathrm{C}_{40} \mathrm{H}_{82}$ \\
51 & Tetracontane & 54.508 & 563 & 0.14 & $\mathrm{C}_{40} \mathrm{H}_{82}$ \\
\hline
\end{tabular}

Table 3. Extracted organic compounds by furfural.

\begin{tabular}{clccc}
\hline P. No. & \multicolumn{1}{c}{ Compound Name } & Rt & Erea\% & M. wt \\
\hline 2 & Ethylcyclohexone & 5.517 & 0.10 & 112 \\
3 & 1,1,3 Trimethyl cyclohexane & 5.608 & 0.11 & 126 \\
5 & 3,3-Dimethy, butylbenzene & 6.667 & 0.42 & 162 \\
9 & IH-, Octahydro-cis Indene & 8.172 & 0.12 & 124 \\
10 & Propyl cyclohexane & 8.533 & 0.28 & 126 \\
12 & 6 Methyl-(E)3-undecene & 8.917 & 0.14 & 168 \\
13 & 1-Pentadecyne & 9.058 & 0.05 & 208 \\
14 & 1- Ethyl-2,3 dimethyl cyclohexone & 9.208 & 0.04 & 140 \\
20 & 3-Cyclohexayl-propnol & 10.483 & 0.10 & 142 \\
21 & Ethyl propyl cyclopentane & 10.567 & 0.11 & 140 \\
26 & Pentyl cyclopentane & 12.100 & 0.11 & 140 \\
28 & Deahydro-trans Naphthalene & 12.708 & 0.12 & 138 \\
29 & Octane, 2,4,6 trimethyl & 12.825 & 0.27 & 156 \\
33 & Methyl(1-methyl Ethyl)benzene & 13.808 & 0.47 & 134 \\
35 & Decahydro-2-methyl Naphthalene & 14.558 & 0.25 & 152 \\
\hline
\end{tabular}




\section{Continued}

\begin{tabular}{|c|c|c|c|c|}
\hline 36 & 2-Butyl1-Octanol & 14.767 & 0.37 & 186 \\
\hline 37 & 1,2,3,4-Tetramethyl Benzene & 14.950 & 0.16 & 134 \\
\hline 40 & Hexyl Cyclopentone & 15.458 & 0.17 & 154 \\
\hline 41 & 2,3 Dyhydro-4-methyl Indene & 15.892 & 0.15 & 132 \\
\hline 42 & 2-Ethyl Hexanoyl chloride & 15.950 & 0.42 & 162 \\
\hline 43 & 2,3,6,7-Ttetramethyl Octane & 16.108 & 0.34 & 170 \\
\hline 44 & 2-Methyl undecon & 16.250 & 0.54 & 170 \\
\hline 45 & 3-Methyl Undecon & 16.450 & 0.35 & 170 \\
\hline 46 & 1-Methyl-2-pentyl Cyclohexane & 16.942 & 0.38 & 168 \\
\hline 47 & 1-(Cyclohexyl methyl)-4 methylas Cyclohexane & 17.075 & 0.23 & 194 \\
\hline 48 & 2.4.4.6-Tetramethyl-1-phenyl-2-heptene & 17.267 & 0.14 & 230 \\
\hline 51 & 1,2,3,4 Tetrahydro-2-methyl Naphthalene & 17.950 & 0.32 & 146 \\
\hline 52 & 10-Heneicosene $(c, t)$ & 18.058 & 0.08 & 294 \\
\hline 53 & 1,2,3,4, Tetrahydro-1-methyl Naphthalene & 18.225 & 0.28 & 146 \\
\hline 54 & Cyclohexane, hexyl & 18.542 & 0.45 & 168 \\
\hline 55 & 3-Tetradecen, (E) & 18.633 & 0.19 & 196 \\
\hline 56 & 6-Methyl Dodecame & 18.933 & 0.11 & 184 \\
\hline 57 & 1-Ethyl-2,3 dihydro-1-methyl-1H-Indere & 18.992 & 0.21 & 160 \\
\hline 58 & 4-Methyl Dodecane & 19.125 & 0.13 & 184 \\
\hline 59 & 2,10-Dimethyl Undecane & 19.267 & 0.42 & 184 \\
\hline 60 & 1,2,3,4- Tetrahydro-5-methyl Naphthalene & 19.458 & 0.57 & 146 \\
\hline 62 & 3,7,11,15, Tetramethyl1-Hexadecanol & 19.717 & 0.20 & 298 \\
\hline 63 & Hepta deoylester octanoic acid & 19.875 & 0.08 & 382 \\
\hline 64 & 2,3-Dihydro-4,7 dimethyl I H-Indene & 19.942 & 0.09 & 146 \\
\hline 65 & 3-Isobutyl-1-methyl-cyclopentane & 20.025 & 0.20 & 154 \\
\hline 67 & Dodecane & 20.833 & 0.32 & 170 \\
\hline 68 & 1,2,3,4-Tetrahydro-2,6 dimethyl Naphthalene & 21.008 & 0.51 & 160 \\
\hline 69 & (2,2-Dimethyl-1-methylenepropyl) Benzene & 21.125 & 0.11 & 160 \\
\hline 70 & 1-Ethyl-2,3-dihydro-1-methyl1H-Indere & 21.342 & 0.10 & 160 \\
\hline 71 & 3,5-Dimethyl dodcane & 21.433 & 0.13 & 198 \\
\hline 72 & 1,1-(1,4-butonediyl) Cyclohexane & 21.567 & 0.39 & 222 \\
\hline 73 & Decylcyclopentane & 21.633 & 0.13 & 210 \\
\hline 74 & 6-Methyl Tridecane & 21.750 & 0.34 & 198 \\
\hline 75 & 5-Methyl Tridecane & 21.825 & 0.19 & 212 \\
\hline 76 & Octadecanoic acid, 2-oxo-methyl ester & 21.867 & 0.28 & 312 \\
\hline
\end{tabular}




\section{Continued}

\begin{tabular}{|c|c|c|c|c|}
\hline 78 & 3,5-Dimethyl dodecane & 22.300 & 0.32 & 198 \\
\hline 79 & 2,7,10-Trimethyl Dodecan & 22.467 & 0.81 & 212 \\
\hline 80 & 17-Chloro7-Heptadecene & 22.908 & 0.37 & 272 \\
\hline 82 & 2,6,10-Trimethyl pentadecane & 23.225 & 0.27 & 254 \\
\hline 83 & Octahydro 2,2,9,4,7,7-hexamethyl-1H-Indene & 23.883 & 0.28 & 208 \\
\hline 84 & Methyl phenyl pentenal & 23.958 & 0.14 & 174 \\
\hline 85 & 4(4-Propyl bicyclohexyl) methyl benzene & 24.192 & 0.23 & 298 \\
\hline 86 & 2-Cyclohexyl Dodecane & 24.417 & 0.81 & 252 \\
\hline 87 & 4-Methyl tetradecan & 24.650 & 0.33 & 212 \\
\hline 89 & 2-N-Butyl-8-N-Hexyl daphthalene & 24.875 & 0.31 & 278 \\
\hline 90 & 3-Methyl tetra decane & 24.975 & 0.23 & 212 \\
\hline 91 & Octa hydro-2,2,4,4,7,7-hexamethyl-trans1H-Indene & 25.133 & 0.36 & 208 \\
\hline 93 & Nonadecane & 25.958 & 5.00 & 268 \\
\hline 94 & 1-Methyl-(6-ethyl-3-decyloxy)-1-silecyclohexane & 26.533 & 0.13 & 298 \\
\hline 95 & Di-tert-dodecyl Disulfide & 26.650 & 0.12 & 402 \\
\hline 96 & Bicyclo (4.1.0) heptan-2-01,1-phenyl endol & 26.767 & 0.11 & 188 \\
\hline 97 & 5-(2-Methyl propyl) Nonane & 26.900 & 0.11 & 184 \\
\hline 98 & Undocane, 4-cyclohexyl & 27.083 & 0.14 & 238 \\
\hline 99 & 4-Methyl Tetradecane & 27.183 & 0.42 & 212 \\
\hline 102 & 3-Methyl Pentadecane & 27.500 & 0.11 & 226 \\
\hline 103 & (R)-(-)-(2)-14-methyl-8-hexa dece-1-ol & 27.600 & 0.25 & 254 \\
\hline 104 & 1-One, 4-methyl, 4-phenyl2-cyclohexen & 27.725 & 0.13 & 186 \\
\hline 105 & 3,3,24-Trimethyl tetracontane & 28.008 & 0.10 & 604 \\
\hline 107 & 3,6-Dimethyl Undcoane & 29.367 & 5.20 & 184 \\
\hline 108 & 3-Cyclohexyl-3cyclohexyl Dodecane & 29.592 & 0.58 & 252 \\
\hline 110 & 3-Methyl Hexa decane & 29.892 & 0.32 & 240 \\
\hline 113 & 9-Octyl Heptadecane & 31.983 & 0.98 & 352 \\
\hline 114 & 3-Methyl Heptadecane & 32.167 & 0.31 & 254 \\
\hline 117 & Hexadecane & 34.150 & 0.65 & 226 \\
\hline 120 & 4-Cyclohxyl Tridecane & 36.383 & 0.26 & 266 \\
\hline 124 & Undecylcyclohexane & 40.383 & 0.25 & 238 \\
\hline
\end{tabular}


Table 4. Physicochemical properties of used petrodiesel sample before and after treatment.

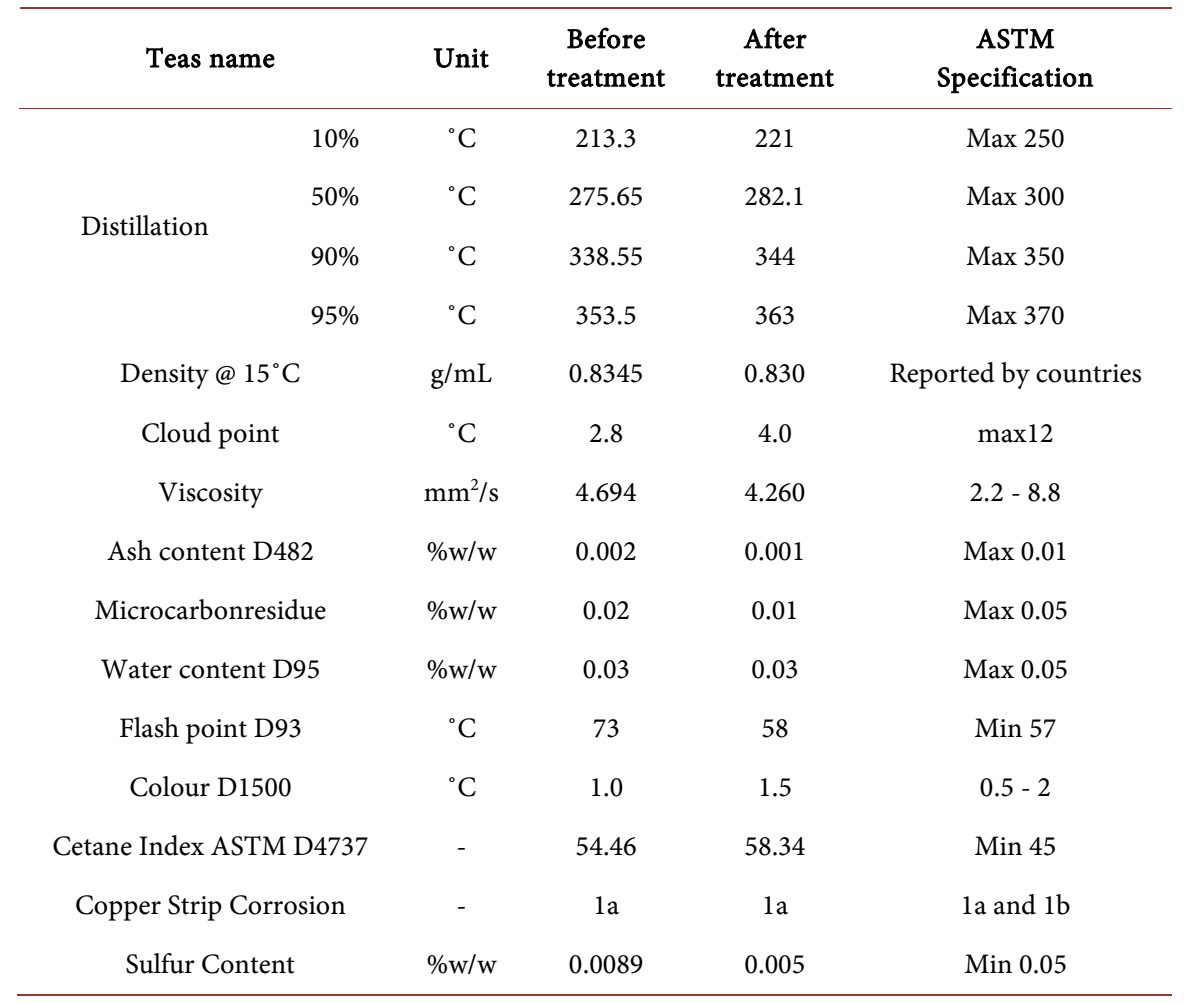

No limits had been assigned for the density by ASTM, because they depend to a greater extent on the temperature prevailing in the country [15] [16] [17] The density has been decreased. Results were found to be within the Khartoum Refinery limit to operate diesel engines [18].

The viscosity of Sudanese petrodiesel sample was decreased after treatment; this result confirmed the quality improvement of petrodiesel fuel.

The decrease of flash point temperature was indicative of overall delay of flammability and hazard [15]. Cloud point was increased to $5.0^{\circ} \mathrm{C}$.

The colour of Sudanese petrodiesel is within ASTM specification [16] although has become intense, this result can be attributed to the brown colour of furfural.

Increase of ash content in diesel may lead to decrease of quality because it may precipitate in engine tank. The ash content of Sudanese petrodiesel sample has been decreased from $0.02 \%$ to $0.01 \% \mathrm{w} / \mathrm{w}$.

Sulfur content has been decreased from $0.0089 \%$ to $0.005 \%$. Showing the efficacy of furfural in abstracting sulfur compounds such as mercaptans, cyclo mercaptanes, thioether and cyclothioethers from used Sudanese petrodiesel, the treatment has no effected on copper strip corrosion property. The cetane number of petrodiesel sample has increased from 54.46 to 58.34 , because the aromatic, cyclo, and branched hydrocarbons were removed by furfural.

Other physicochemical such as distillation, micro carbon residue and water content properties were improved by furfural and their results have been found 
within ASTM specification.

\subsection{Elements Composition of Petrodiesel before and after Treatment by Furfural}

The data depicted in Table 5 has revealed that the petrodiesel metal concentrations of $\mathrm{As}, \mathrm{Ca}$ and $\mathrm{Fe}$ were the highest. It has shown slight decrease after improvement. A result which might have been the cause to decrease the ash content [19]. While some metal concentrations remained unchanged.

Furfural is an important renewable, non-petroleum based feedstock. It has acted as a selective solvent in refining petrodiesel fuel. Aromatics, cyclic and branched hydrocarbons were extracted and removed from petrodiesel by means of furfural extraction. Cetane number has increased without the decomposition of furfural, hence furfural can act as a biobased alternative to the thermally unstable 2-ethylhexyl nitrate.

\section{Conclusions}

Based on the previous discussion, conclusion can be summarized:

- The organic compounds of Sudanese petrodiesel sample have been evaluated by using Gas Chromatography Mass Spectrometry GC/MS technique, before and after treatment by furfural.

- A total of 81 organic compounds have been extracted from Sudanese petrodiesel sample. Most of the extracted compounds are aromatic, cyclic, and branched organic compounds.

- The physicochemical Characteristics of Sudanese Petrodiesel sample have been investigated before and after treatment by furfural according to American Society for Testing and Materials, characteristic include: density, viscosity, flash point, colour, cloud point, water content, ash content, micro carbon residue, copper strip corrosion, sulfur content and cetane number.

Table 5. Elements composition of sudanese petrodiesel before and after treatment by furfural.

\begin{tabular}{ccc}
\hline Element & Before Treatment $(\mathrm{ppm})$ & After Treatment $(\mathrm{ppm})$ \\
\hline $\mathrm{Na}$ & 0.433 & 0.432 \\
$\mathrm{Mg}$ & 0.278 & 0.258 \\
$\mathrm{Ca}$ & 2.055 & 2.005 \\
$\mathrm{~V}$ & 0.1 & 0.1 \\
$\mathrm{Fe}$ & 1.062 & 0.895 \\
$\mathrm{Ni}$ & 0.1 & 0.1 \\
$\mathrm{Cu}$ & 0.1 & 0.1 \\
$\mathrm{Al}$ & 2.636 & 2.615 \\
$\mathrm{As}$ & 0.142 & 0.1 \\
$\mathrm{~Pb}$ & 0.1 & 0.1 \\
\hline
\end{tabular}


- The furfural has shown a slight intensity on colour of Sudanese Petrodiesel but all other results were within permissible limits assigned by ASTM.

- The elements composition of Sudanese petrodiesel sample has been performed by Inductively Coupled Plasma Technique.

- The concentrations of $\mathrm{Na}, \mathrm{Mg}, \mathrm{Ca}, \mathrm{Fe}, \mathrm{Al}$ and As have been slightly decreased after treatment by furfural, these results led to decrease of ash content.

- Furfural has proved to be an efficient non-expensive, sulfur compound adsorbent and thermal stable cetane number improver for the quality improvement of Sudanese petrodiesel fuel.

\section{References}

[1] Ezeldin, M. and Ishak, C.Y. (2016) Characterization of Physiochemical Properties of Sudanese Petrodiesel Samples Produced from Khartoum Refinery in Sudan. The Journal of Organic Chemistry, 98, 42512-42517.

[2] Davis, A.M.J. and Brenner, H. (2001) The Falling-Needle Viscometer. Physics of Fluids, 13, 3086-3088. https://doi.org/10.1063/1.1398537

[3] Duncan, A.M., et al. (2012) High-Pressure Viscosity of Soybean-Oil-Based Biodiesel Blends with Ultra-Low-Sulfur Diesel Fuel. Energy \& Fuels, 26, 7023-7036. https://doi.org/10.1021/ef3012068

[4] Duncan, A.M., et al. (2010) High-Pressure Viscosity of Diesel from Soybean, Canola, and Canola Oils. Energy \& Fuels, 24, 5708-5716. https://doi.org/10.1021/ef100382f

[5] Gruse, W.A. and Stevens, D.R. (1960) Chemical Technology of Petroleum. 3rd Edition, McGraw-Hill Book Company, New York, 42.

[6] Harris, K.R., Kanakubo, M. and Woolf, L.A. (2007) Temperature and Pressure Dependence of the Viscosity of the Ionic Liquids 1-Hexyl-3-Methylimidazolium Hexafluorophosphate and 1-Butyl-3-Methylimidazolium Bis(Trifluoromethylsulfonyl)imide. Journal of Chemical \& Engineering Data, 52, 1080-1085. https://doi.org/10.1021/je700032n

[7] Isdale, J. (1976) Cetane Number of Simple Liquids Including Measurement and Prediction at Elevated Pressure. Ph.D. Thesis, University of Strathclyde, Glasgow, UK.

[8] Annual Book of ASTM Standards (2005) American Society for Testing and Materials. Salvter. J. Rand, West Conshohocken.

[9] Lee, S.W., et al. (2002) Effects of Diesel Fuel Characteristics on Spray and Combustion in a Diesel Engine. JSAE Review, 23, 407-414.

[10] Park, N.A. and Irvine Jr., T.F. (1984) The Falling Needle Viscometer a New Technique for Viscosity Measurements. Wärme-und Stoffübertragung, 18, 201-206. https://doi.org/10.1007/BF01007130

[11] Riazi, M.R. and Al-Otaibi, G.N. (2000) Estimation of Viscosity of Liquid Hydrocarbon Systems. Fuel, 80, 27-32.

[12] Yamaki, Y., et al. (2001) Heavy Duty Diesel Engine with Common Rail Type Fuel Injection Systems. Japanese Society of Automotive Engineers, Tokyo, Japan.

[13] Vanleenawen, J.J., Jonkery, R.J. (1994) Octane Number Production Based on Gas Chromatography Analysis with Non Linear Regression Techniques. Chemometrics and Intelligent Laboratory Systems, 24, 325-345. https://doi.org/10.1016/0169-7439(94)85051-8 
[14] Ezeldin, M., Masaad, A.M., Abualreish, M.J.A. and Osama, A. (2015) Physico-Chemical Properties of Blended Gasoline Samples produced from Khartoum Refinery in Sudan. Research Journal of Chemistry and Environment, 19, 22-31.

[15] Ezeldin, M., Elamin, A.A., Masaad, A.M., Suleman, N.M. and Osama, A.A. (2015) Effect of X-Ray Radiation on Some Physicochemical Characteristics of Diesel Fuel. American Research Thoughts, 1, 2862-2870.

[16] Ezeldin, M., Masaad, A.M., Suleman, N.M. and Abualreish, M.J.A. (2015) Effect of Diethyleamine on Some Physicochemical Properties of Reformat Gasoline. American Journal of Scientific Research, 6, 88-96.

[17] Ezeldin, M., Nasir, S.A.G., Masaad, A.M. and Suleman, N.M. (2015) Determination of Some Heavy Metals in Raw Petroleum Wastewater Samples Before and After Passing on Australis Phragmites Plant. American Journal of Environmental Protection, 4, 354-357.

[18] Moh, E. and Massad, A. (2015) Quality Improvement of Sudanese Gasoline by Using Di Isopropyl Ether and Moringa Oil. European Academic Research, 3, 2748 2763.

[19] Jadallh, A.A. and Ezeldin, M. (2016) Effect of Synthetic Zeolite on Some Physical Characteristics and Research Octane Number of Final Product Gasoline Sample Produced from Khartoum Refinery in Sudan. American Chemical Science Journal, 13, 1-6. https://doi.org/10.9734/ACSJ/2016/22788

\section{Submit or recommend next manuscript to SCIRP and we will provide best} service for you:

Accepting pre-submission inquiries through Email, Facebook, LinkedIn, Twitter, etc. A wide selection of journals (inclusive of 9 subjects, more than 200 journals)

Providing 24-hour high-quality service

User-friendly online submission system

Fair and swift peer-review system

Efficient typesetting and proofreading procedure

Display of the result of downloads and visits, as well as the number of cited articles

Maximum dissemination of your research work

Submit your manuscript at: http://papersubmission.scirp.org/

Or contact ajac@scirp.org 